\title{
A SURVEY OF T TAURI STARS WITH AKARI
}

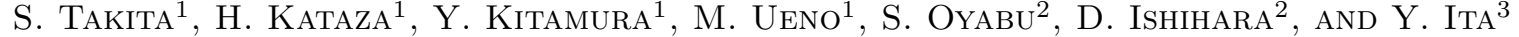 \\ ${ }^{1}$ Institute of Space and Astronautical Science, Japan Aerospace Exploration Agency, 3-1-1 Yoshinodai, Chuo, \\ Sagamihara, Kanagawa 252-5210, Japan \\ ${ }^{2}$ Graduate School of Science, Nagoya University, Furo-cho, Chikusa-ku, Nagoya, Aichi 464-8602, Japan \\ ${ }^{3}$ Astronomical Institute, Graduate School of Science, Tohoku University, 6-3 Aramaki Aoba, Aoba-ku, Sendai, \\ Miyagi 980-8578, Japan \\ E-mail: takita@ir.isas.jaxa.jp \\ (Received June 30, 2012; Accepted July 31, 2012)
}

\begin{abstract}
We have carried out a survey of T Tauri stars (TTSs) in a 1,800-square-degrees region toward the Taurus-Auriga star forming region with the AKARI Mid-Infrared All-Sky Survey. By combination of AKARI, 2MASS, and UCAC surveys, we created new criteria to chose TTS candidates. We also considered Asymptotic Giant Branch stars and galaxies, which have similar infrared colors, to separate TTSs from these sources. On the basis of our criteria, we find 27 new TTS candidates. To verify our criteria, we performed follow-up observations for them and confirmed that 23 are TTSs.
\end{abstract}

Key words: stars: formation; stars: late-type; stars: pre-main sequence; infrared: stars

\section{INTRODUCTION}

T Tauri stars (TTSs) are low-mass pre-main sequence stars with ages of 1-10 Myrs and own circumstellar disks which are thought to be the birthplace of planets. Most of TTSs have been found near their natal molecular clouds (size of $\sim 10 \mathrm{pc}$ ). On the other hand, it is now known that a substantial fraction of TTSs are located far away from molecular clouds (e.g., Wichmann et al., 1996). These TTSs are thought to be born in small $(\sim 0.1 \mathrm{pc})$ clouds, and the natal clouds are dissipated. This situation indicates that many TTSs are thought to be still unexplored. Therefore, an unbiased survey of TTSs is required in order to understand star and planet formation processes. For this purpose, we used AKARI All-Sky Survey to increase the number of solitary TTSs. We chose the Taurus-Auriga region, which is a well-studied region of low-mass star formation at a distance of $140 \mathrm{pc}$, as a testbench because hundreds of TTSs are known and thus, we were able to estimate typical colors of TTSs.

\section{SOURCE SELECTION}

We have developed criteria to select TTS candidates by combining the AKARI MIR Point Source Catalogue (PSC), the 2MASS PSC, and the 3rd USNO CCD Astrograph Catalog (UCAC3). We used several colorcolor and color-magnitude diagrams to choose IR excess sources as TTS candidates. However, since AGB stars and galaxies also have IR excess emission, we also considered them.

At first, since AGB stars have hot (typically $>100 \mathrm{~K}$ ) dust while TTSs have cold (typically $<100 \mathrm{~K}$ ) one, we can separate AGB stars using $\operatorname{MIR}(S 9 W-L 18 W)$ colors. Second, galaxies and the central stars of PNe are faint at visible wavelengths, so we can remove them using the brightness at visible wavelengths. Finally, we choose sources with excess emission from $J-K_{S}$ vs. $K_{S}-S 9 W$ and $J-K_{S}$ vs. $K_{S}-L 18 W$ colorcolor diagrams which correspond to colors of previously known TTSs, as TTS candidate sources. The details of the criteria are described in Takita et al. (2010). 
On the basis of our criteria, we selected 176 sources out of 14725 AKARI sources, which are distributed in the $2^{h} 40^{m}<\mathrm{RA}<5^{h} 40^{m}$ and $0^{\circ}<$ Dec $<40^{\circ}$ area. We searched these 176 sources in the SIMBAD database, and found that 27 sources have no identifications or literatures, so we considered them as new TTS candidates.

\section{FOLLOW-UP OBSERVATIONS}

We obtained follow-up spectroscopic observations at Kitt Peak National Observatory (KPNO) for our 27 new TTS candidates. The objectives were to find $\mathrm{H} \alpha$ emission/absorption line $(6,563 \AA)$ and LiI absorption line $(6,707 \AA)$. Since $\mathrm{Li}$ is easily destroyed when the stars begin nuclear fusion, the existence of $\mathrm{Li}$ is the most important feature of young stars. Usually, the equivalent width of $\mathrm{Li}(\mathrm{W}(\mathrm{Li}))$ of the Pleiades members are used as Zero-Age-Main-Sequence (ZAMS) stars, and the typical value is $0.1 \AA$. We performed intermediate resolution $(R \sim 3,000)$ spectroscopy at the optical wavelengths of $6,000-7,000 \AA$ using the Gold Camera on the 2.1 meter telescope.

Among the 27 target stars, we have detected significant $\mathrm{Li}$ absorption lines in 23 sources with $\mathrm{W}(\mathrm{Li})$ $\gtrsim 0.1 \AA$; we have thus 23 new TTSs. Although we were able to see $\mathrm{Li}$ in 2 other sources, they are weak $(\ll 0.1 \AA)$, and we could not detect $\mathrm{Li}$ in the remaining 2 sources. Of the 23 new TTSs, 18 sources have $\mathrm{H} \alpha$ emission line, and the other 5 sources have absorption lines. We classified Classical TTSs (CTTSs: $\sim 1 \mathrm{Myr}$ ) and Weak-line TTSs (WTTSs: $\sim 10 \mathrm{Myr}$ ) by full width at $10 \%$ of the $\mathrm{H} \alpha$ emission profile peak. White \& Basri (2003) proposed that the full width at $10 \%$ of $>270 \mathrm{~km} \mathrm{~s}^{-1}$ indicates the presence of an accretion disk (i.e., CTTS). We fitted a Gaussian to the emission line and measured the $10 \%$ width, and got 15 CTTSs and 8 WTTSs. There are 9 CTTSs and 6 WTTSs which are located far ( $>2 \mathrm{deg}$ or $>5 \mathrm{pc}$ at distance of $140 \mathrm{pc}$ ) away from molecular clouds (see Figure 1). The natal clouds of these TTSs are thought to be small ( $\sim 0.1 \mathrm{pc})$ and thus already have been dissipated.

\section{SUMMARY}

We constructed new criteria to extract TTS candidates by using AKARI (MIR) as well as UCAC (optical) and 2MASS (NIR) surveys, which are valid for the whole sky. We performed a survey of TTSs toward a 1,800square-degree region of Taurus-Auriga star forming re-

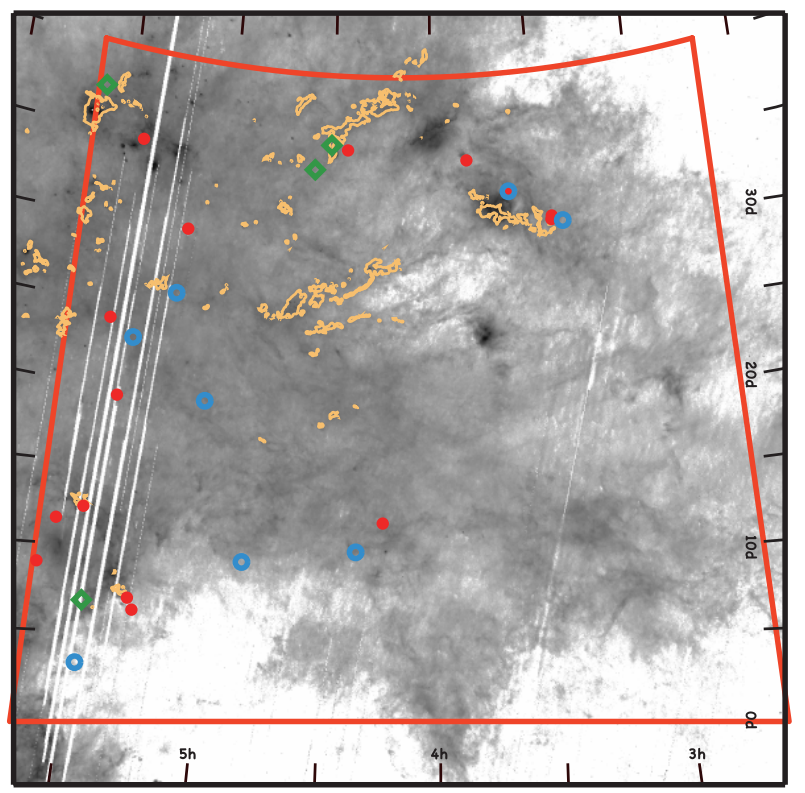

Fig. 1. The spacial distribution of the new TTSs. The red filled circles and cyan open ones are CTTSs and WTTSs, respectively. The green diamonds were candidate sources, but turned out not to be TTSs after the follow-up observations. The grey scale is AKARI $90 \mu \mathrm{m}$ map, and the contours represent the extinction of $A_{V}=2$ (Dobashi et al., 2005).

gion, and got 27 new TTS candidates. We performed follow-up spectroscopic observations at optical wavelengths, and found that 23 sources are TTSs. This result indicates the usefulness of our method. From the line width of the $\mathrm{H} \alpha$ emission, we identified 15 CTTSs and 8 WTTSs. The existence of 15 solitary TTSs (9 CTTSs and 6 WTTSs) indicates that the star formation in small clouds is universal.

\section{ACKNOWLEDGEMENTS}

This work is based on observations with AKARI, a JAXA project with the participation of ESA.

\section{REFERENCES}

Ishihara, D., et al., 2010, The AKARI/IRC MidInfrared All-Sky Survey, A\&A, 514, A1

Ita, Y., et al., 2010, AKARI's Infrared View on Nearby Stars, A\&A, 514, A2

Takita, S., et al., 2010, A Survey of T Tauri Stars with AKARI Towards the Taurus-Auriga Region, A\&A, 519, A83 